\title{
A Case Report of Primary Pulmonary Choriocarcinoma in a Man: Successful Combination of Surgery and Chemotherapy
}

\author{
Hang Thi Thuy Nguyen ${ }^{a}$ Hung Huy Hoang ${ }^{a}$ Anh Thi Van Le ${ }^{b}$ \\ aDepartment of Medical Oncology 2, Vietnam National Cancer Hospital, Hanoi, Vietnam; \\ ${ }^{b}$ Department of Oncology, Hanoi Medical University, Hanoi, Vietnam
}

\author{
Keywords \\ Choriocarcinoma $\cdot$ Primary pulmonary choriocarcinoma $\cdot$ Man $\cdot$ Human chorionic \\ gonadotropin
}

\section{Abstract}

Choriocarcinoma is a malignant tumor that typically appears in gonadal organs and primarily occurs in women of reproductive age. Being a primary extragonadal choriocarcinoma, primary pulmonary choriocarcinoma (PPC) is an extremely rare condition. Due to the rarity of PPC, no standardized treatment has been established so far. However, surgery combined with adjuvant chemotherapy appears to be the most optimal treatment. Here, we report a rare case of a man with PPC that was successfully treated with surgery followed by chemotherapy.

\section{Introduction}

Choriocarcinoma is classified as a germ cell tumor consisting of syncytiotrophoblastic cells and able to produce human chorionic gonadotropin (hCG) hormone [1]. Although primary gonadal choriocarcinomas commonly metastasize to the lungs (45-87\%), primary pulmonary choriocarcinoma (PPC) is a very rare condition [2]. Unlike gestational choriocar- 
cinoma, which responds well to chemotherapy and has a good prognosis even when detected at a late stage, PPC has a very poor prognosis with a dismal 5-year survival rate of $<5 \%$ [3]. PPCs progress rapidly and mostly disseminate to the bone, liver, brain, spleen, and contralateral lung [4]. To diagnose PPC, the exclusion of gonadal neoplasms, $\beta$-hCG-positive tumors in the lung, and confirmation on histopathology are required.

Due to the paucity of data related to PPC, there is no official guideline for treatment of this disease. Nevertheless, several studies have shown that the combination of surgery and chemotherapy represents a favorable treatment [5]. In this report, we present a male patient with PPC successfully treated with surgery followed by chemotherapy.

\section{Case Presentation}

A 42-year-old man was referred to us complaining of unrelenting right-sided chest pain and 3-kg weight loss over 2 months. The patient had a 30-pack-year smoking history. Physical examination including the lungs, heart, peripheral lymph nodes, abdomen, and testes was normal. No abnormality in other organs was detected. A chest computed tomography scan (CT scan) revealed an $82 \times 92 \mathrm{~mm}$ mass in the right upper lobe with an ill-defined margin, little enhancement, and visceral pleural involvement. The residual parenchyma had no abnormality. Enlarged lower paratracheal and subcarinal lymph nodes (stations 4R, 7) were also detected on the CT scan, with the biggest dimension being $11 \mathrm{~mm}$. Bronchoscopy was subsequently performed and showed a tumor in the right upper lobe which was histopathologically defined as a squamous carcinoma (small specimen).

Other screenings including CT of the abdomen, pelvis, and brain as well as bone scintigraphy showed no abnormality. Tumor markers measured were Cyfra $21-1(16.48 \mathrm{ng} / \mathrm{mL})$ and carcinoembryonic antigen $(2.9 \mathrm{ng} / \mathrm{mL})$. Thus, the patient was initially diagnosed with stage cT3N1M0 non-small cell lung cancer and underwent radical surgery including tumor resection and systematic lymph node dissection. Intraoperative assessment showed a $10 \times 15 \mathrm{~cm}$ tumor involving the wall of the superior vena cava, the phrenic nerve, and the subclavian artery. The surgeon dissected the tumor away from the vessels and nerves to avoid damaging them. Then he exposed the total tumor and definitively resected the lesion.

Postoperative pathology showed choriocarcinoma, with the mediastinal lymph nodes all negative. To confirm the definite diagnosis, immunohistochemical (IHC) staining was subsequently conducted. The results affirmed that this was a choriocarcinoma, with CK7 $(+)$, CKAE1/AE3(+), CK20(-), TTF-1(-), napsin A(-), p40(+), SALL4(+), HCG(+), Plap(-), and glypican 3(-) (Fig. 1).

Serum $\beta$-hCG after the operation was 2,134 mIU/mL. Preoperative assessment showed the only tumor in the right lung without any lesions in gonads. Therefore, the patient was diagnosed with localized-stage PPC and underwent a debulking surgery. Because of extension of the tumor and the involvement of vessels and nerves, the postoperative status was evaluated to achieve an $\mathrm{R} 1$ resection (microscopic residual tumor).

One month after the operation, the patient was reassessed by chest CT. The scan detected a lesion progressing at the initial site of the primary tumor. In detail, the screening revealed a $24 \times 30 \mathrm{~mm}$ tumor in the right upper lung adjacent to the right subclavian vessels and a 3 -mm nodule in the left upper lung with an enlarged subcarinal lymph node of $11 \times 18 \mathrm{~mm}$. The rapid progression of the disease was consistent with the PPC's characteristics $[3,4]$.

After a multidisciplinary discussion, we allocated adjuvant chemotherapy with an EMA-CO regimen to the patient. Reassessment was performed after 4 cycles of chemotherapy, and it might have been considered to imply adjuvant radiation therapy if there were residual lesions in the thorax.

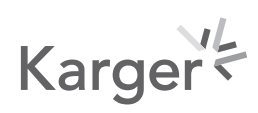




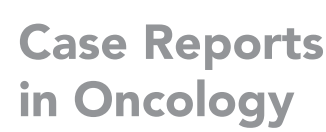

\begin{tabular}{l|l}
\hline Case Rep Oncol 2020;13:923-928 \\
\hline DOI: 10.1159/000508744 & $\begin{array}{l}\text { ○ 2020 The Author(s). Published by S. Karger AG, Basel } \\
\text { www.karger.com/cro }\end{array}$ \\
\hline
\end{tabular}

Nguyen et al.: Primary Pulmonary Choriocarcinoma in a Man: A Rare Case
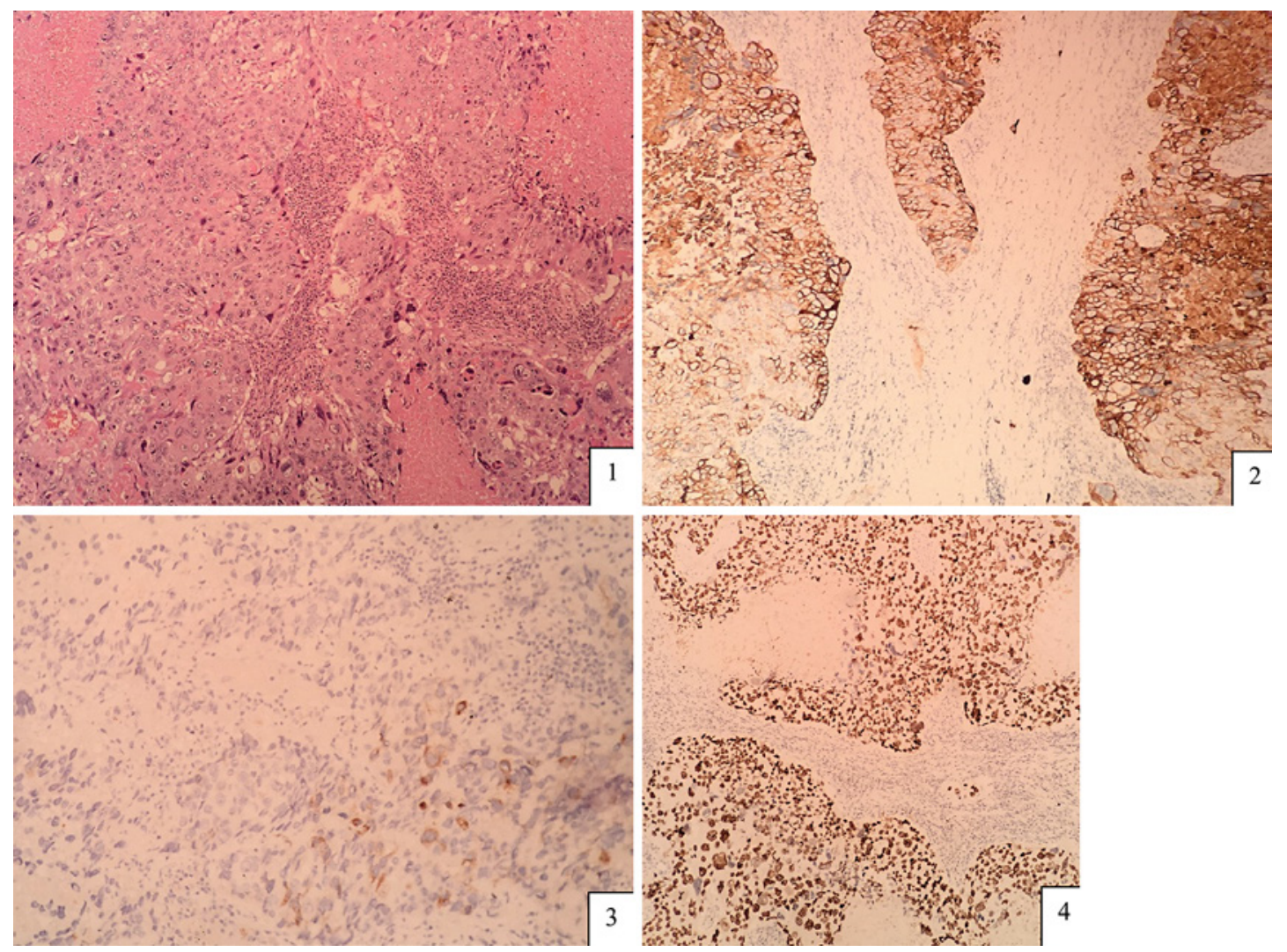

Fig. 1. 1 Histopathological feature of the tumor. 2 Immunohistochemistry with CK7 (+). $3 \beta$-hCG(+). 4 SALL4(+).

Two cycles of the EMA-CO regimen the patient tolerated and responded to very well. Chest CT did not detect any lesions in the lungs and the mediastinum. Serum $\beta$-hCG was reduced to $<0.1 \mathrm{mIU} / \mathrm{mL}$.

The assessment after 4 cycles of chemotherapy showed a complete response; there were no tumors discovered in the lungs by chest CT and $\beta$-hCG remained normal. The patient was discharged and initiated a post-treatment follow-up. Currently, after 6 months, there has been no recurrence detected (Fig. 2).

\section{Discussion}

Choriocarcinoma is a highly malignant tumor derived from trophoblastic cells. Due to the affinity with blood vessels of these cells, choriocarcinomas frequently disseminate through a hematogenous route and most commonly metastasize to the lungs [6]. Primary extragenital choriocarcinomas mainly originate in the retroperitoneum, mediastinum, or intracranium. However, they rarely arise in the ovary, stomach, bladder, and lungs [7]. Despite the limited knowledge about the cell origin and pathogenesis of PPC, several theories have been established: (1) primordial germ cells that migrate to the lung during embryogenesis; (2) highgrade transformation from a non-trophoblastic lung neoplasm to a PPC; or (3) PPC and giant cell carcinoma in the lung are the same entity. Although these theories are all reasonable, it 


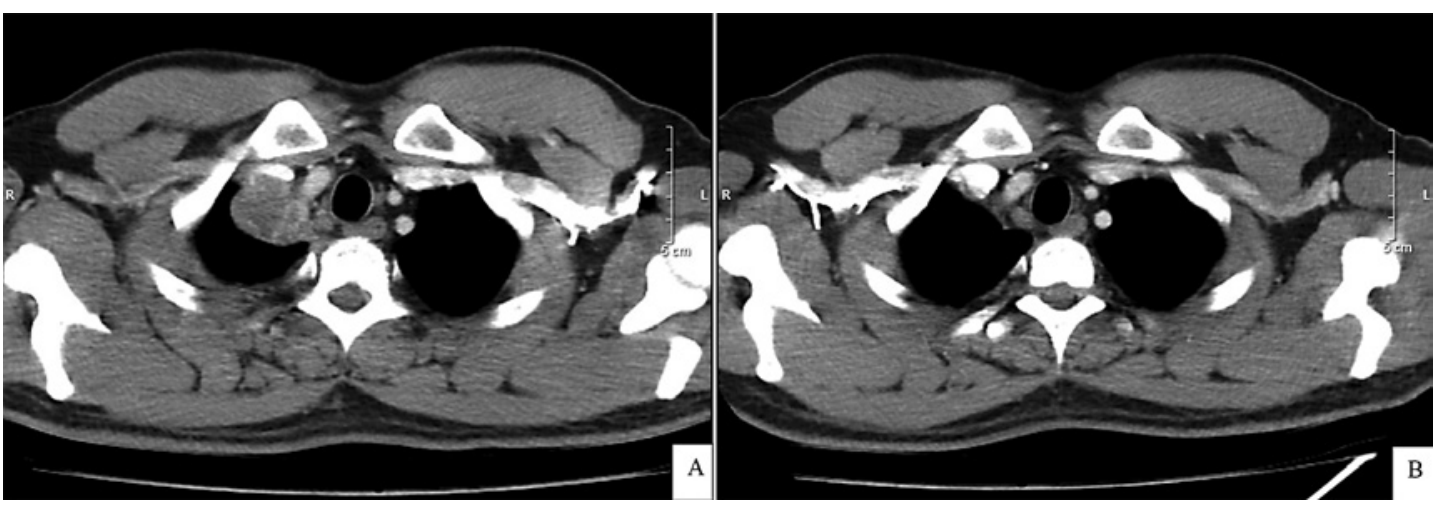

Fig. 2. Chest CT scans. A One month after the operation. B After 2 cycles of chemotherapy.

is necessary to make further assessments of its molecular biology to provide useful evidence for this rare malignancy [8].

\section{Diagnosis}

The clinical presentations of PPC are similar to those of many other lung cancers; they include respiratory symptoms (cough, chest pain, and dyspnea), symptoms due to compression or invasion of the tumor (dysphagia and superior vena cava syndrome), etc. In our case, the patient on admission presented with chest pain, and chest CT revealed a tumor in the right lung extending to the mediastinum. Biopsy through bronchoscopy was performed to take out a small specimen, which was preliminarily diagnosed as a squamous carcinoma. Therefore, the initial diagnosis was non-small cell lung cancer stage IIIA. The patient was assigned to surgery, and postoperative pathology showed choriocarcinoma.

The diagnostic criteria for PPC include the following: (1) history of no gynecologic cancers; (2) isolated or predominant lesion detected in the lung after ruling out primary lesions in gonads; ( 3 ) high levels of $\beta$-hCG that return to normal after surgery or chemotherapy; and (4) the diagnosis of PPC is confirmed by pathology [3, 5].

The most important differential diagnosis of PPC is $\beta$-hCG-producing giant cell carcinoma, which has similar morphologic features to PPC. Despite secreting $\beta$-hCG, hCG-producing giant cell carcinoma is strongly immunoreactive to TTF-1, which is an immunohistochemical marker of epithelial origin in the lung. By contrast, TTF-1 is negative in PPC [9]. Our patient had hCG(+) and TTF-1(-) on IHC staining, which means the PPC diagnosis was most reasonable.

Choriocarcinomas commonly derive from gonads, and the majority metastasize to the lung. Thus, the diagnosis of PPC needs to be made cautiously after excluding all gonadal lesions and other sites that choriocarcinoma may arise from $[2,10]$.

In our case, CT of the chest, abdomen, and pelvis, along with testicular ultrasound, showed no abnormality except for the sole lesion in the right lung. The patient also did not have genitourinary symptoms. Postoperative $\beta$-hCG was still elevated due to the residual tumor, but it quickly decreased just after 2 cycles of chemotherapy. Postoperative pathology and IHC staining confirmed choriocarcinoma. Therefore, the definite diagnosis for this patient was PPC.

\section{Treatment}

The operation just achieved an R1 resection because the extended tumor adhered to other crucial organs in the mediastinum and the surgeon could only remove the visible lesion as much as possible. The residual lesion progressed rapidly, expanding to a size of $3 \mathrm{~cm}$ with a 2-cm mediastinal lymph node after only 1 month. 
Given that PPC is an extremely rare occurrence, there is no standard guideline for the treatment of this disease. Due to its undifferentiated characteristics, PPC poorly responds to radiation therapy [5]. Hence, surgery combined with chemotherapy appears to be the most appropriate management. Several options for the chemotherapeutic treatment of PPC can be used, such as BEP and EMA-CO [11]. Though there have been no randomized trials proving its superiority, the EMA-CO regimen is usually selected as the initial treatment for high-risk gestational trophoblastic disease in most countries [12].

Poor prognostic factors according to Ziga et al. [5] include male gender, age $>40$ years, smoking, tumor size $\geq 5 \mathrm{~cm}$, metastasis, and history of non-gestation. The patient in our report had several poor prognostic factors, being male and having an age of 42 years, a history of smoking, and an extended tumor with a size of $10 \times 15 \mathrm{~cm}$.

Also in the study by Ziga et al. [5], patients treated with surgery followed by chemotherapy derived a greater benefit in terms of survival than those who were treated with surgery alone or chemotherapy alone. Therefore, chemotherapy following surgery can be considered necessary. Because the patient in our report had multiple poor prognostic factors, the most appropriate regimen was EMA-CO (etoposide, methotrexate, actinomycin D, cyclophosphamide, and vincristine). After the consultation, we consensually agreed to add 4 cycles of the EMA-CO regimen, and a response evaluation would be performed after every 2 cycles.

After 2 cycles of chemotherapy, the patient was reassessed with screenings and $\beta$-hCG. The results showed that the patient had responded completely and no lesions were detected on chest CT. The level of serum $\beta$-hCG was also reduced to normal. This was in accordance with a PPC setting.

After 4 cycles, the patient remained stable, the lesions in the lungs and mediastinum had completely disappeared, and $\beta$-hCG was normal. The patient was discharged and followed up every 3 months. Currently, the patient has remained disease free after the 6-month follow-up.

\section{Conclusion}

PPC is a rare disease and its pathogenesis is unclear. Giant cell carcinoma, which is classified as a malignant epithelial tumor of the lung, can be mistaken for PPC. Thus, IHC staining, $\beta$-hCG measurement, and examination to exclude primary gonadal malignancies are crucial to give a definite diagnosis. Although PPC has a poor prognosis with rapid progression, it effectively responds to chemotherapy. There is no standard treatment recommendation at present. The optimal choice that is preferred is a combination of surgery and chemotherapy for patients with localized-stage PPC.

\section{Acknowledgements}

We greatly acknowledge the staff of the Department of Medical Oncology 2, Dr. Khac Kiem Nguyen of the Department of Thoracic Surgery, and Dr. Thi Tuoi Tran of the Department of Pathology, Vietnam National Cancer Hospital, for their help with our patient.

\section{Statement of Ethics}

The patient has given written informed consent to publish his case (including publication of images). The study protocol was approved by the ethics committee of Vietnam National Cancer Hospital.

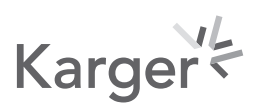




\section{Conflict of Interest Statement}

The authors have no conflicts of interest to declare.

\section{Funding Sources}

Funding for the publication of this article is provided by AstraZeneca Vietnam Company Limited.

\section{Author Contributions}

H.T.T. Nguyen: main doctor, revised the manuscript; H.H. Hoang: followed up the patient, revised the manuscript; A.T.V. Le: assistant surgeon, wrote the manuscript.

\section{References}

1 Di Crescenzo V, Laperuta P, Napolitano F, Carlomagno C, Garzi A, Vitale M. An unusual case of primary choriocarcinoma of the lung. BMC Surg. 2013;13(Suppl 2):S33.

2 Vegh GL, Szigetvári I, Soltesz I, Major K, Batorfi J, Dancso J, et al. Primary pulmonary choriocarcinoma: a case report. J Reprod Med. 2008;53:369-72.

3 Serno J, Zeppernick F, Jäkel J, Schrading S, Maass N, Meihold-Heerlein I, et al. Primary pulmonary choriocarcinoma: case report and review of the literature. Gynecol Obstet Invest. 2012;74(2):171-6.

4 Kini U, Babu MK. Primary pulmonary choriocarcinoma: is it still an enigma? Indian J Chest Dis Allied Sci. 2007; 49:119-226.

5 Snoj Z, Kocijancic I, Skof E. Primary pulmonary choriocarcinoma. Radiol Oncol. 2017;51(1):1-7.

6 Li XM, Liu XY, Liu ZX. Choriocarcinoma with multiple lung, skull and skin metastases in a postmenopausal female: a case report. Oncol Lett. 2015;10(6):3837-9.

7 Tanimura A, Natsuyama H, Kawano M, Tanimura Y, Tanaka T, Kitazono M. Primary choriocarcinoma of the lung. Hum Pathol. 1985;16:1281-4.

8 Rali P, Xie J, Rali G, Rali M, Silverman J, Malik K. A rare case of metastatic choriocarcinoma of lung origin. Case Rep Pulmonol. 2017;2017:4649813.

9 Kamata S, Sakurada A, Sato N, Noda M, Okada Y. A case of primary pulmonary choriocarcinoma successfully treated by surgery. Gen Thorac Cardiovasc Surg. 2016;65(6):361-4.

10 Rhee YK, Kim JH, Kim WH, Ha CY, You KH, Jang DS. Primary choriocarcinoma of the lung. Korean J Intern Med. 1987;2(2):269-72.

11 Maestá I, Leite FV, Michelin OC, Rogatto SR. Primary pulmonary choriocarcinoma after human chorionic gonadotropin normalization following hydatidiform mole: a report of two cases. J Reprod Med. 2010;55: 311-6.

12 Lurain JR, Singh DK, Schink JC. Primary treatment of metastatic high-risk gestational trophoblastic neoplasia with EMA-CO chemotherapy. J Reprod Med. 2006;51:767-72.

\section{Karger'}

